\title{
La noción de justicia en sociedades democráticas: \\ una mirada desde Paul Ricoeur
}

\author{
The notion of justice in democratic societies: \\ a view from Paul Ricoeur
}

\author{
ARiel Rosales Úbeda \\ Trabajador social UC, Magíster en Trabajo Social UC. Docente de la Escuela de Trabajo Social de la Universidad San Sebastián, \\ Concepción, Chile. Correo electrónico: aarosalesu@gmail.com
}

\begin{abstract}
Resumen
Este artículo tendrá como principal eje la noción de justicia desde el pensamiento hermenéutico de Paul Ricoeur en el marco de lo que el mismo autor denomina el mal político de la sociedad y su expresión "la tragedia de lo social". De este modo, frente a la demanda por más ética de las sociedades contemporáneas se intenta presentar la visión de Ricoeur como una nueva posibilidad dentro del debate acerca de lo justo. Se trabaja la idea de justicia y sus aspectos hermenéuticos más relevantes para contribuir a este debate en sociedades plurales.
\end{abstract}

Palabras clave. justicia, deliberación, ética.

\begin{abstract}
Summary
This article focuses on the concept of justice from Paul Ricoeur's hermeneutical thinking in the context of what the author calls the political evil of society and its expression as "social tragedy". Thus, in attention to the demand for more ethics in contemporary societies the attempt of this article is to present Ricoeur's vision as a new possibility in the debate about the just. It works the idea of justice and its most relevant hermeneutical aspects to contribute to this debate in plural societies.
\end{abstract}

Key words. justice, deliberation, ethics, Ricoeur.

\section{Introducción}

Dentro de sociedades democráticas, "el mal" constituye una de las preocupaciones filosóficas y políticas más importantes de los últimos años. El carácter paradójico de lo político muestra, por una parte, que "la existencia política del hombre desarrolla un tipo de racionalidad específica, irreductible a la dialéctica de base económica"; y, por otra, que la sociedad "desarrolla males específicos que, son precisamente males políticos, males asociados al poder político" (Ricoeur, 1990, p. 261). Esta es la doble y específica originalidad de lo político sobre la cual la noción de justicia se va constituyendo en las sociedades contemporáneas.

En este escenario, se levanta el desafío de explicitar la problemática del mal en la sociedad e iluminar la paradoja que ello representa (Ricoeur, 1990, p. 261-262). Aquí interesa especialmente reflexionar sobre cómo la política, el Estado y la sociedad no pueden ocultar la conflictividad que se presenta en sus relaciones y que se expresa en -no pocas- violencias (la violencia producto de la diferencia social, la violencia impuesta por la exigencia del orden, etc.) que pueden ser consideradas como injusticias. De este modo, la preocupación por el mal político, incentiva a recuperar la problemática de la justicia dentro de sociedades caracterizadas por la diferenciación y la pluralidad. De ahí que nos enfrentamos a la cuestión de lo justo y de lo injusto en un plano donde la reflexión sobre la justicia se conjuga con la historicidad de las situaciones sociales a las cuales interpela.

El debate acerca de lo justo en sociedades contemporáneas se enfrenta a la creciente diferenciación entre los ideales de vida buena de los individuos, lo cual coloca un marco en que la ciudadanía se cruza cada vez más con la idea de la afirmación de la diferencia y la promoción de la diversidad. En este sentido, el análisis de las ciencias sociales, hoy 
en día se enfrenta a prácticas que son definidas por sujetos colectivos fuera de la esfera laboral y territorial, y que entran en la esfera de la subjetividad política; las cuales son politizadas y llevadas a la lucha por el respeto de derechos y compromisos. Así, las diferencias de género, etnia, orientación sexual, consumo de bienes, minorías, etc.; son las identidades narrativas que hoy en día emergen ante las problemáticas derivadas del no-reconocimiento y que requieren de la mediación de la justicia para su expresión (Ricoeur, 1996). Para ser todavía más precisos, la tragedia social que se presenta en nuestras sociedades, refiere a la situación en la que una variedad de pensamientos, visiones de mundo y explicaciones de la realidad cohabitan en un territorio, sin que ninguna de estas visiones pueda tener hegemonía sobre otra; y donde la noción de justicia puede ser tan plural como las identidades narrativas que la reclaman.

Esta diversidad surge de una condicionante básica: se presenta al interior de una sociedad "abierta" o "sociedad libre", en la que se exige la actualización de las premisas de la justicia que sustentan la convivencia ciudadana. Al mismo tiempo, la apertura social que refiere al ejercicio de la libertad dentro de un conjunto social, sugiere el desenvolvimiento de los principios morales de cada sujeto al interior del orden social (Ladriere, 1997). Así, una sociedad es moralmente pluralista cuando en ella conviven distintas concepciones de vida buena, así como diversas concepciones de hombre; cuando ante la pregunta sobre cómo podemos ser felices existen distintas respuestas.

En dicho escenario, se requiere repensar los fundamentos ético-morales que sustentan la idea de justicia a partir de la cual se gesta la convivencia entre diversas identidades culturales atomizadas en las sociedades modernas. Ahora bien, el camino de la reflexión ética nos llevaría a un camino diverso de perspectivas filosóficas cuyo eje fundamental versa sobre la noción de lo justo y lo polisémico de su definición. De este modo, y frente a la polisemia de la noción de justicia, variados autores (Rawls, Habermas, Cortina, Mill, entre otros.) han desarrollado propuestas teóricas para enfrentar las situaciones de injusticia de nuestras sociedades. Sin embargo, y para efectos de este trabajo, se tratará el tema de la justicia desde la mirada hermenéutica de Paul Ricoeur, para con ello contribuir al debate acerca de lo justo en sociedades contemporáneas.

En este artículo, y bajo el objeto de dilucidar la noción de justicia en la ética aplicada de Ricoeur, se muestra un camino para salir del debate entre dos tradiciones que históricamente han sido puestas en conflicto en la discusión acerca de lo justo en nuestra sociedad, a saber: teleologismo y deontologismo. La vía propuesta asume el desafío de repensar los fundamentos éticos de la justicia en base a modos deliberativos que capturen y pongan a la luz las orientaciones de vida buena de los actores sociales en contextos de interacción social -con y para otros- y dentro del ordenamiento institucional que requieren -y exigen- las democracias actuales. Desde luego, y como se ha señalado, Ricoeur no es el único filósofo que se ha ocupado de abordar las tensiones entre lo bueno y lo justo. Sin embargo, lo que distingue a este autor es que no pretende privilegiar una perspectiva por sobre otra, sino que más bien su innovación está en presentar la noción de lo justo en base a la complementariedad entre ambas perspectivas. En este sentido, el sujeto de la filosofía de Ricoeur es un ser concreto y capaz que establece relaciones éticas de solicitud y cuidado con el otro cercano y lejano-, en el plano de lo particular, pero que del mismo modo está relacionado y obligado con el cada uno de la sociedad -universo de todos los demás-, y por ello se requiere de una mediación institucional que haga frente a la desigualdad y el conflicto.

El planteamiento de este trabajo indica que la justicia surge de un cruce entre ética y moral, entre los ideales de vida buena de los ciudadanos -ética- y las normas universales que rigen el orden societal -moral-, siendo lo segundo la expresión de lo primero. Dicho de otro modo, la comprensión de los ideales de vida buena de los actores haría posible la realización de las aspiraciones de los sujetos que comparten contextos diversos a partir de mediaciones institucionales adecuadas a cada situación de injusticia.

La estructura de este artículo se muestra en base a argumentos en los cuales se distinguen ciertos aspectos que permiten comprender la configuración de la noción de justicia en la ética aplicada de Paul Ricoeur. Con esto se pretende aportar a la discusión de lo justo en las sociedades contemporáneas.

\section{La estructura de la moralidad y la justicia en Ricoeur}

A partir de la publicación de La teoría de la Justicia de J. Rawls, Paul Ricoeur inicialmente se fue centrando en la dimensión teleológica de la acción, situándose en un principio del lado de las éticas del bien frente a las éticas procedimentales de orientación kantiana. Sin embargo, y con el avance de sus postulados, el autor desarrolla una articulación de 
ambas perspectivas para referirse a la noción justicia en las sociedades contemporáneas.

Desde la mirada de Ricoeur, y bajo el amparo de la fenomenología-hermenéutica del sí mismo -donde el yo y el bien se constituyen mutuamente-, la noción de justicia propuesta se basa en la estructura de la moralidad que desarrolla Ricoeur en "su pequeña ética", y que admite dos visiones -una horizontal y otra vertical-complementarias.

La primera de dichas visiones constituye el sí mismo del sujeto, a quien su deseo de vivir una vida buena le impulsa a comprender que dicha vida ha de ser vivida, con y para los otros, y que para ello ha de contar con la mediación de instituciones justas. En este proceso, el sí mismo se constituiría dialécticamente mediante la mediación de la alteridad, pues, para Ricoeur, en el origen de las relaciones interpersonales está el reconocimiento del otro como lejano, como un tercero impersonal cuya validez es tan originaria como la propia. Así, el sí mismo se apertura a la totalidad de los seres humanos bajo el signo de la solidaridad.

A partir de la tríada sí mismo-otro-institucionalidad, Ricoeur parece tomar en cuenta los conceptos de cuidado de sí, cuidado del otro, y cuidado de la institución. El primero de dichos términos refiere a la autonomía (entendida como autoestima), la cual hace alusión a la capacidad de obrar de manera intencionada y a la capacidad de iniciativa para introducir cambios en la dinámica de la vida. Ahora bien, para hablar de autonomía es necesario ponerla en relación con la demanda de reciprocidad que fuerza al sujeto a atribuir al otro las mismas capacidades que descubre en sí mismo. Esto da paso a un segundo momento marcado por el signo de la solicitud. Por último, la vida en sociedad obliga a la apertura del sujeto hacia las instituciones, a las que -entendidas como sistema de reparto- se les exige el tránsito de la solicitud a la justicia, lo cual lleva consigo una exigencia de igualdad y de universalidad distinta a la amistad presente en las relaciones cercanas

Por otra parte, en la visión vertical de la estructura de la moralidad en Ricoeur, encontramos una relación de subordinación y complementariedad entre los puntos de vista teleológico, deontológico y prudencial. El enfoque teleológico, guiado por la idea de "vivir bien", es complementario y al mismo tiempo subordina al deontológico, donde imperan la norma y el procedimiento. La trayectoria que toma este eje señala que el carácter teleológico de la acción humana atraviesa el plano de la norma y termina en el nivel de la sabiduría práctica, al que Ricoeur llama "prudencia", entendida -desde un matiz aristotélico- como el arte de la decisión equitativa en situaciones de incertidumbre y de conflicto. El primero y el tercero de estos puntos constituyen lo que Ricoeur llama la ética, y el segundo e intermedio, la moral. De este modo, considera que la justicia es la regla práctica más elevada por constituir el punto donde termina el deseo de vivir bien y, a la vez -mediada por la equidad-, representa el último tramo del enfoque vertical donde prima la sabiduría práctica. Aunque la idea de "bueno" hace que la justicia dependa originariamente del deseo de vivir bien, es la noción de "lo justo" la que, al poner en movimiento la dialéctica de la moral (horizontal y vertical), sitúa a la bondad bajo el signo de la prudencia.

Conforme a esto, es posible señalar que el concepto de lo que entiende Ricoeur aquí por justicia refiere a la operación de la sabiduría práctica y a la toma de decisiones dentro de procesos deliberativos en las correspondientes instituciones que ordenan la vida en sociedad. Esto muestra que la noción de justicia parte de la consideración de las situaciones particulares que aquejan a los actores sociales, y fundamentalmente de los ideales de vida buena de los mismos. En consecuencia, Ricoeur cumple la tarea de vincular la ética del deseo, la moral del deber, y la sabiduría práctica en el plano de la razón práctica.

\section{La noción de justicia en Ricoeur más allá de lo procedimental}

Al entrar en la discusión acerca de la justicia, es posible observar que Ricoeur elimina de esta noción sus elementos más kantianos -el énfasis constructivista- para colocar en primer plano la noción de "equilibrio reflexivo" entre el deseo y la norma. Así, el referirse a la justicia no se trata de situarse únicamente bajo una regla general, sino también de interpretar los hechos ocurridos y la norma en función de saber en qué medida lo deliberado "se ajusta" a los hechos, lo cual exige la adecuación de la norma al caso.

Ahora bien, al pensar lo justo en las sociedades actuales, generalmente se piensa en la conformidad con la norma y el aparato legislativo. La idea de conformidad con la ley va a significar la adecuación de las acciones con las leyes nacidas de la actividad legislativa del cuerpo político. Esto señala que, en las sociedades contemporáneas, lo justo se asocia al formalismo procedimental que caracteriza a los sistemas jurídicos y políticos occidentales, donde se ha pasado de "un apriorismo riguroso a un positi- 
vismo jurídico donde la cuestión del fundamento cae bajo el control de las instancias legisladoras empírica e históricamente constituidas bajo fundamentos únicamente deontológicos" (Ricoeur, 1995: p. 182-183), quedando fuera las expectativas y las formas de vida de quienes son obligados por la norma. Dicho de otro modo el positivismo jurídico de las democracias occidentales excluye la historicidad de las situaciones en las cuales se aplica la norma, lo que puede ser considerado como fuente de injusticia en la sociedad.

Todas estas cuestiones relevan la insuficiencia que, para Ricoeur, presenta una concepción puramente procedimental de la justicia, la cual no tiene en cuenta la diversidad cualitativa de las situaciones en las que se apela a ella. En este sentido, Ricoeur presenta a la justicia como una idea que va más allá de lo procedimental, y con ello se refiere al modo de operación de la justicia, y presenta una serie de elementos que se articulan entre sí para conformar una idea de lo justo que está entre lo bueno y lo legal. En este sentido, la justicia refiere a la historicidad social y a las relaciones entre seres humanos y las instituciones.

Bajo el impulso de la tradición kantiana, la teoría de la justicia se ha vuelto hacia el lado deontológico, esto es, a una concepción en la que todas las relaciones morales, jurídicas y políticas están situadas bajo la idea de la conformidad con la ley, de la legalidad; lo cual parece insuficiente en el marco de la creciente policontextualidad social. En esta reflexión, se vuelve relevante la tesis de Paul Ricoeur, la cual señala que una concepción únicamente procedimental de la justicia refiere -en el mejor de los casos- a la racionalización de un sentido de la justicia que ya está supuesto en los agentes, y refuerza por tanto la idea de que lo bueno es anterior a lo justo.

Para Ricoeur, los procedimientos racionalmente estructurados permiten, en un primer momento, distinguir qué normas de las surgidas en el mundo de la vida -siguiendo a Habermas- son correctas. Así, en primera instancia, la función de estos procedimientos es la de actualizar el concepto kantiano de "voluntad racional", esto es, actualizar lo que todos, bajo determinadas condiciones podrían querer, y que por su propia condición de voluntad racional asume el carácter de universal. En este sentido, en el procedimentalismo los contenidos concretos exceden el campo de la ética, entendida bajo un matiz teleológico, pues corresponden a los mundos de la vida. Así, siguiendo a Cortina, "el procedimentalismo asigna a la ética únicamente la tarea de descubrir los procedimientos legitimadores de las normas" (Cortina, 2000: p. 75-78). Para Ricoeur, el procedimentalismo intenta dar razones de la pretensión de universalidad de la moral y por ello apela a estructuras cognitivas y procedimientos que exhiben la universalidad más allá de los contenidos concretos de la existencia, lo que muestra un carácter deontológico fundamental alejado de los ideales y situaciones particulares de existencia de los agentes sociales. Así, los procedimientos legitimadores de la justicia pueden describirse sin depender para ello de los diversos contextos, y pretenden por tanto justificar-ilusoriamente- su universalidad.

Sumado a lo anterior, se puede agregar que la no consideración de las particularidades contextuales excluye de las consideraciones normativas de las decisiones políticas las consecuencias de las acciones, quedando solo un aspecto normativo asociado a universalidad de la ley donde se considera a todos por igual sin la consideración de las diferencias humanas (Ricoeur, 2001). Así, el apegarse a la norma parece más importante que las preocupaciones y expectativas existenciales de los individuos socializados en relación a sus vidas individuales y colectivas.

Con esto Ricoeur emprende la tarea de mostrar la parcialidad y la insuficiencia tanto del procedimentalismo como del deontologismo para referirse a la justicia. Insuficiencia marcada por la pretensión de eliminar la ética -orientación a la felicidad-en los análisis sociales, esto es: el desvincular la moral de la fase teleológica que la precede, y del plano de la sabiduría práctica donde se realiza.

Según lo señalado más arriba y frente a la imposibilidad de entender "lo justo" independientemente del bien, es posible señalar que, en términos concretos, el anhelo de vivir bien es un componente fundamental de la justicia (Ricoeur, 2000). De esta manera, se observa que el remarcar la idea de que la justicia "no es puramente moral sino que tiene también un significado ético", indica que "lo justo se sitúa entre lo legal y lo bueno" y que por ello, "el sentido de la justicia no se agota en la construcción de los sistemas jurídicos que suscita" (Ricoeur, 1995, p. 177-178). De este modo, para Ricoeur, la idea de lo justo -sea en el sentido o en el contenido- rige las prácticas sociales de toda índole, por lo que importa destacar que la justicia se plantea como un giro que va desde lo bueno a lo obligatorio.

Según lo dicho, parece que la propuesta postprocedimentalista -por llamarla de ese modo- de Ricoeur, trata de retomar la crítica proveniente de los comunitaristas como Michael Sandel (1998), 
Charles Taylor (1985), Michael Walzer (1983), y Alasdair MacIntyre (1984), entre otros, acerca de la noción de justicia en sociedades plurales, donde la noción de justicia no puede entenderse como un pacto "a-histórico", como el relacionado a la posición original bajo el velo de ignorancia rawlsiano.

\section{Justicia, historicidad y espacio público}

En su obra Lo Justo (1997), Ricoeur plantea que lo político aparece como el entorno por excelencia para el logro de la justicia. El autor menciona que sin la mediación institucional, el individuo solo sería "un bosquejo de hombre"; es por ello que su pertenencia a un cuerpo político es necesaria para su desarrollo humano y para la expresión de la ciudadanía. De esta manera la participación de los ciudadanos en los asuntos públicos no es digna de ser anulada. Al respecto señala que: "el ciudadano surgido de la mediación institucional no puede sino anhelar que todos los humanos gocen como él de esta mediación política que, sumándose a las condiciones necesarias para el sujeto capaz, devienen en condiciones suficientes para el ciudadano real" (Ricoeur, 1997: p. 37).

En este marco, la capacidad humana que requiere la justicia se desarrolla en lo que Ricoeur -siguiendo a Hannah Arendt- denomina "el espacio público de aparición”. Aquí la noción de espacio público expresa la condición de pluralidad que resulta de la extensión de las relaciones interpersonales de los agentes sociales, y a su vez, esta condición de pluralidad se caracteriza por el anhelo de vivir juntos de una comunidad histórica cuya expresión es la institucionalidad política. Con esto se quiere decir que la efectuación de la libertad individual dentro de una historia común de valores y el reconocimiento de la libertad de la segunda persona, pasa por la "justa" mediación de la institucionalidad frente e los males presentes en la sociedad.

Ahora bien, frente a la pregunta por los valores éticos que se exigen en este nivel político de la institución, Ricoeur indica sin muchos rodeos, y siguiendo a Rawls, que es la justicia la primera virtud de las instituciones y que por lo tanto es este principio el que rige el orden social. En este marco, la justicia va más a allá de las relaciones cara a cara y se refiere a cada cual en cuanto tercero al interior de espacios públicos en donde el ser humano aparece. Ricoeur señala a lo largo de su obra sobre la justicia que el valor que asuma la noción de justicia tiene que ver con la historicidad de cada situación en la cual se apela a lo justo. En este sentido, lo que él llama "justicia", tiene que ver con el valor de la memoria, tanto personal como colectiva. En este marco, el olvido del "otro" y de su "historicidad" aparece como fuente de injusticias.

La historicidad de la justicia no es accidental, sino que corresponde a un rasgo profundo de la vida social que se trata precisamente de esclarecer en el ejercicio de la sabiduría práctica que opera en el proceso de definición de lo justo, es decir, en la deliberación. Así por ejemplo, la deliberación respecto a lo justo de la distribución de los bienes de la sociedad se presenta como un momento develador de la historicidad de la justicia; y ocupa así, un lugar intermedio entre lo universal y las modalidades concretas de la vida social de los actores (Ricoeur, 1996, p. 231-242). De este modo, no se trata de saber si son las demandas del individuo o de la comunidad las que deben prevalecer, sino más bien de saber si los principios de justicia que rigen la estructura de la sociedad responden a las convicciones éticas adoptadas por los ciudadanos.

Con lo dicho parece que la noción de justicia refiere a un juicio en tiempo presente cuyo insumo se relaciona a la memoria -a la historia- de los agentes sociales que la reclaman para sí mismos y para los otros a las instituciones públicas. En este sentido, la justicia deja de ser un concepto atemporal y se entiende más bien como una noción histórica que requiere permanente actualización. Así, la emergencia -histórica- de la noción de justicia está estrechamente relacionada con la evaluación de las relaciones sociales y las formas objetivas en las cuales esas relaciones se institucionalizan. Por ejemplo, la noción de derecho a la participación social y la política de participación ciudadana en Chile no puede comprenderse sino en una sociedad capaz de crear exclusión. Así, toda forma que asume la justicia tiene relación con la historicidad de los problemas a los cuales intenta responder. En este sentido, la justicia presenta un matiz de veracidad asociado al contexto en el cual se despliega la solicitud. En este sentido, "la memoria encuentra el sentido de la justicia en el camino de la crítica histórica" (Ricoeur, 2000, p. 635).

De esta manera puede verse que Ricoeur no deslinda la idea de la justicia de la sabiduría práctica que opera en las situaciones concretas del marco deontológico de la obligación, en el cual lo justo se identifica con lo legal (Ricoeur, 1995). Más concretamente, el autor sitúa a la justicia en el marco histórico del Estado de derecho y de la protección de los derechos en situaciones concretas (Ricoeur, 1993). 


\section{Los componentes y las dimensiones de la noción de justicia en Ricoeur}

Ricoeur observa que, en un primer momento, los sujetos presentan un sentido de lo justo en relación a lo que consideran injusto, lo cual está asociado al reclamo y la queja; así es que lo justo refiere al olvido de ejercer la equidad en distintas situaciones. De este modo, "al parecer los hombres tienen una visión más clara de lo que falta en las relaciones humanas que del modo recto de organizarlas" (Ricoeur, 2001; p. 231). Así, frente a la fijación de la injusticia al interior de la sociedad, la noción de justicia hace referencia a los problemas del orden público y a los bienes que allí se reúnen que, en sociedades democráticas, requieren de mediación institucional. Una de las razones que hace necesaria la mediación institucional de la justicia es que el deseo de vida buena de los individuos lleva siempre a la desigualdad, por cuanto, la vida social es siempre desigual en cuanto a la pluralidad de las orientaciones de vida y a la distribución de los bienes disponibles en la sociedad.

Cuando el aparato de las instituciones media para el cumplimiento de la justicia se observa la presencia de algunas dimensiones que pueden expresarse en legislaciones, leyes, políticas públicas, entre otros mecanismos, para compensar las injusticias. Así, al tratar la paradoja de lo político, Ricoeur, desde la perspectiva de la fundamentación filosófico-política de lo justo, muestra que la noción de justicia presente en la ciudadanía es fundamental para hacer frente a los males políticos que generan injusticia en las sociedades actuales.

Al referirse al modo de operación de la justicia, el autor observa que en la práctica, la justicia utiliza los símbolos de la espada y la balanza; lo cual implica que la noción de justicia presenta una tendencia hacia el castigo y hacia la equidad de manera simultánea. No obstante este modo de operación de la justicia, Ricoeur presenta una serie de elementos que se articulan entre sí para conformar una idea de la justicia que está entre lo bueno y lo legal. Analíticamente, la noción de justicia a la cual se hace referencia presenta una serie de componentes asociados a su carácter dialógico (reconocimiento y reciprocidad), y además una diferenciación interna que abarca dos dimensiones que se pueden distinguir en las prácticas humanas. Por un lado, apela al derecho y a la noción jurídica de la justicia -uso de la espada- donde aparece la dimensión restaurativa de la justicia y los conceptos de pena y rehabilitación. Por otro, refiere -de modo más extenso- a la justicia bajo el signo de la equidad, la cual va más allá de lo jurídico y se expande a todo ámbito de la razón práctica. Aquí aparece la dimensión constructiva bajo la forma del reparto, donde señala el carácter distributivo de la justicia, tema que abarca la mayor parte de su trabajo acerca de la justicia en las sociedades actuales.

\section{Reconocimiento, reciprocidad y justicia}

Frente al mal político y a la incompletitud de la democracia en las sociedades contemporáneas, Ricoeur pone el acento en el modo en que el reconocimiento jurídico amplía la esfera de los derechos reconocidos (para cada individuo y para nuevas categorías de personas) y, al mismo tiempo, enriquece la gama de capacidades que los sujetos se reconocen entre sí (Ricoeur, 2005). Así también subraya el modo en que la distancia entre la distribución de los derechos (por ejemplo sociales y económicos) y la distribución de los bienes crea un "contraste insoportable" que es una fuente central de la injusticia (Ricoeur, 2005, p. 208). Con todo, Ricoeur pretende evitar el riesgo de la anulación de los sujetos bajo un supuesto tecnicismo instrumental para tratar los asuntos sociales y la alteridad.

En esta línea, Ricoeur sostiene que la justicia, ahora asociada a la idea reconocimiento, no hay que buscarla necesariamente mediante la lucha - a diferencia de Honneth (1997)-, pues esta conlleva siempre un juego de fuerzas cuya lógica comporta la posibilidad de la intensificación hasta los límites de la violencia, y por ende de la inmoralidad; sino que más bien "hay que buscarla en experiencias pacificadas de reconocimiento mutuo, que descansan en mediaciones simbólicas sustraídas tanto al orden jurídico como al de los intercambios comerciales" (De la Maza, 2010, p. 25). Esta tesis coloca el modelo de la reciprocidad de los intercambios justos, que tiende a borrar el carácter interpersonal de los intercambios basados en el amor. En este sentido, Ricoeur señala que los intercambios justos serían aquellos en los que hay equivalencia y proporcionalidad, sin embargo, también plantea que para que haya equivalencia se exige comparación y cálculo, lo que posibilita la aparición de nuevos conflictos en torno a los criterios del cálculo y la adecuación -justeza- de su aplicación (Ricoeur, 2005, p. 95).

Para Ricoeur, el reconocimiento transita de la voz acti$v a$, referida al reconocimiento como identificación, a una voz pasiva, que alude al reconocimiento mutuo, pasando por el reconocimiento de sí mismo. En el polo activo, el reconocimiento expresa la pretensión de ejercer una forma de control intelectual sobre el mundo, en cambio, en el polo pasivo, el 
pedido de reconocimiento manifiesta una expectativa que solo puede ser satisfecha a través de la reciprocidad (Ricoeur, 2005). Es así como la justicia exige reconocimiento mutuo y, con ello, requiere de reciprocidad.

Las relaciones sociales siempre se enmarcan originariamente en una fuerte desigualdad, y es justamente allí donde la justicia reclama la reciprocidad. De este modo, considerar la reciprocidad como exigencia social y como principio de la deliberación pública implica considerar al otro como ser capaz y sufriente en todo proceso de decisión, en cuanto a su experiencia y a su orientación de vida buena. En este sentido, la reciprocidad apunta a la mutualidad entre actores y se opone a la idea liberal de reciprocidad situada por encima de los agentes del intercambio.

Así, bajo el signo de la reciprocidad, se espera que los ideales de vida buena de todo tercero sean reconocidos y aceptados para que así se supere la desigualdad existente en los intercambios sociales. La idea es que la desigualdad pueda ser compensada por una auténtica reciprocidad en el intercambio, para lo cual se requiere de la institucionalidad pública, como un tercero que medie dicha relación de forma neutral. Así, la justicia se encuentra en una constante búsqueda de igualdad moral a través de todos los caminos posibles de reconocimiento.

A partir de la inclusión de las ideas de reconocimiento y de reciprocidad, la justicia exige la integración del "otro" en procesos de deliberación pública. Esto permite que la acción pública resultante sea vista como una respuesta "adecuada" a las situaciones y solicitudes expresadas. En este sentido, la reciprocidad opera como una especie de ofrecimiento que refleja el reconocimiento de los ideales de vida buena de los actores que, siendo reconocidos como sujetos capaces, dan contenido a la decisión. Ahora bien, hay que tener presente que el proceso de distribución de bienes sociales es un proceso conflictivo que opera sobre la base de recursos escasos, los cuales no alcanzan a responder a la totalidad de las demandas de los actores. Esto claramente indica que no toda solicitud entra a los espacios de deliberación y que por lo tanto la discusión acerca de los asuntos públicos es un proceso siempre histórico que invita a actualizar permanentemente las situaciones sociales sobre las cuales se demanda justicia. Lo anterior exige mediar entre fórmulas contextualistas y universalistas ${ }^{1}$, lo cual es un proceso posible -aunque no menos complejo- que implica considerar un principio de universalización como referente, sin dejar de prestar atención al contexto de la acción en donde se interpreta la existencia, ni caer - por exceso- en una suerte de relativismo. De este modo, en las situaciones de aplicación de las normas no se está autorizado a desistir del empleo de la sabiduría práctica.

\section{Justicia restaurativa}

Frente a la presencia de los males políticos y a lo trágico de la vida social, Ricoeur es consciente del hecho de la transgresión de la norma y de la existencia de instancias de castigo para la mantención del orden social. A partir del análisis crítico de las diversas filosofías de fundamentación racional de la justicia y del derecho de punir del Estado, Ricoeur va a plantear "una utopía de la justicia no violenta" cuyo objetivo consiste en ser una justicia reparadora -en el sentido de restauración- o "justicia reconstructiva" como el mismo autor señala (Ricoeur, 1995: p. 44-55).

Al presentar la dimensión reconstructiva de la justicia y su carácter no violento, Ricoeur piensa en humanizar la justicia -fundamentalmente la penala través de procedimientos de reparación del daño hecho por el delincuente y de reincorporación a la vida social. En este sentido, plantea la reparación para la víctima y la restauración de capacidades para el victimario. Se trata de un proyecto de justicia centrado en la reconstrucción o restauración del vínculo social perdido por el agente mediante un acto delictual.

La reflexión de Ricoeur sobre la justicia reconstructiva se cruza con lo que él mismo denomina "el restablecimiento de la paz social" (Ricoeur, 1995). Uno de los motivos fundamentales de su postulado acerca de la justicia reconstructiva está relacionado al compromiso público que se exige a las instituciones en sociedades democráticas frente a los males

1 Respecto a este tema existen dos posiciones que se confrontan. Por un lado encontramos a los "racionalistas-universalistas" que, como Rawls y Habermas, sostienen que la finalidad de la discusión pública es establecer verdades universales válidas para todos e independientes del contexto sociohistórico de los actores. Las implicaciones de la visión universalista indican que la justicia debe apelar a principios generales y que su objetivo debe ser tratar de encontrar fórmulas inclusivas que pueden servir para medir la justicia social en las sociedades modernas. Por otra parte, contextualistas como Michel Walzer y Richard Rorty, niegan la disponibilidad de un punto de vista fuera de las prácticas socio-históricas y de las instituciones sociales de una sociedad determinada a partir de las cuales se podrían hacer juicios universales "contextualmente independientes". En este sentido, lo que hay de contenido en las deliberaciones deriva de los juicios particulares que los actores están inclinados a hacer como participantes de modos de vida especificos (Mouffe, 2003, p. 2-4). 
políticos de las mismas. Aquí la idea es fortalecer la integración real de los agentes y no reproducir situaciones de exclusión derivadas de la transgresión de la norma.

Ricoeur en sus obras Lo Justo (Sanción, Rehabilitación y Perdón) y en Lo Justo 2 (Justicia y Venganza) presenta una reflexión acerca de la pena y del derecho a punir del Estado. La ejecución de la pena, más allá de su duración efectiva, equivale a un proceso de desocialización acelerada que trae consecuencias posteriores en la vida de los sujetos. Para Ricoeur, la exclusión de la vida en sociedad, a expensas de todo proyecto de inserción, engendra de manera progresiva a sujetos reproductores de los males sociales. Así, la noción de sanción, entendida únicamente como castigo, constituye una negación flagrante de toda idea de restauración, y por ello la negación absoluta de todo proyecto de restablecimiento de derechos y la imposibilidad de acercase a una vida buena (Ricoeur, 1995). Por tanto, para nuestro autor la sanción tendrá sentido, sí y solo sí, conduce a la rehabilitación, lo que supone la obligación de reeducar a quien delinque para volver a hacer de él un ciudadano, lo cual pasa por una vuelta al reconocimiento de sus capacidades.

En este marco, el autor considera que la pena y la sanción, más allá de su carácter castigador, ha de ir necesariamente orientada al restablecimiento de los derechos de una persona, de un estatuto jurídico que había perdido, "a restaurar la capacidad del condenado para volver a ser ciudadano pleno al concluir su pena" (Ricoeur, 1995: p. 201).

Ahora bien, la preocupación de Ricoeur por lo injusto y la necesidad de un sentido de justicia no solo se observa en el ámbito de la justicia penal sino también en la esfera de lo político. Esta es una de las claves que permiten despejar el recorrido y la esencia de la obra de Paul Ricoeur. Desde dicha preocupación, la justicia no solo presenta el signo de la espada, sino que también toma el signo de la balanza.

\section{Justicia constructiva}

No obstante el dominio de la noción de justicia por parte de la legalidad y del aparato judicial, Ricoeur, en su condición de filósofo moral, está interesado en explorar otro de los ejes en los que se fundamenta la justicia y que está presente en toda sociedad entendida como sistema de reparto de roles, tareas, beneficios y cargas. Específicamente vislumbra la temática de la justicia "en función de aquellas situaciones ejemplares en las que nuestra indignación se inflamaba contra la injusticia: repartos desiguales, pobreza extrema, desventajas institucionales, pagos excesivos (...) en los que la justicia se anuncia como distribución justa" (Ricoeur, 1990, p. 22). Este nivel implica una estrategia distributiva asociada a "bienes a compartir".

La distribución genera un conflicto cuya base refiere a la interpretación de la importancia de los bienes para cada quien. En esto, a menudo se pierden de vista los elementos cualitativos con los que estos "bienes" están cargados, y que hacen que ellos signifiquen más de lo que a veces son; por lo que en ocasiones se hace imposible trazar una equitativa división de los mismos. De esta manera, la justicia refiere a una norma universal de distribución que dé respuesta a la solicitud -siempre contextualenunciada por los agentes sociales bajo el signo de la equidad. En este sentido, Ricoeur apuesta por mantener abierta la noción de distribución para que el aporte del concepto de "reparto" que está implícito en ella, y que hace falta a la noción de "querer vivir juntos", permita encontrar un pilar adecuado en la idea de justicia.

En un primer momento, lo justo puede ser entendido bajo la noción de reparto aritmético donde se asume la consigna de "dar a cada uno lo suyo" (Ricoeur, 1996, p. 165). Sin embrago, esto puede presentar el sesgo de considerar a la sociedad como un territorio llano y homogéneo en cuanto a las necesidades y deseos de vida buena. De este modo, la aplicación de la regla de justicia a las interacciones humanas requiere no solo considerar a la sociedad como un vasto sistema de reparto de funciones, cargos, y bienes económicos esenciales, sino también como un sistema amplio, diverso y diferenciado en donde se reparte.

Así, la justicia requiere ser corregida en un primer momento por la noción de igualdad aritmética, para luego ser corregida por la igualdad proporcional; pues donde la generalidad de las leyes y sus principios no ofrecen soluciones frente a situaciones dilemáticas, o cuando normas con el mismo peso parecen contradecirse mutuamente, ambas ideas se entrecruzan entregando resultados diversos. Así, la noción de justicia en Ricoeur se atiende bajo la luz de la equidad en tanto adecuación del reparto de los bienes y derechos sociales al interior de un Estado de derecho.

En esta noción de distribución, es posible hacer una distinción entre los términos "participación" y "partición". Al respecto se indica que tener participación es una cosa y otra muy distinta es recibir una parte de algo. No obstante tal diferencia, ambos términos son complementarios y no excluyentes. 
Así, solo los que reciben dentro de una sociedad de cooperación pueden ser considerados como participes de esa sociedad, en la medida en que las partes distribuidas estén co-ordenadas entre sí; esto es lo que podría marcar la distancia entre integración y marginación. En este marco, una institución considerada reguladora de la distribución de funciones solo existe para los individuos que forman parte de esas funciones, y ella será justa o injusta según el modo cómo se aplique la equidad -proporcionalidad- en la distribución.

Desde lo dicho, la justicia, como principio de distribución pública, trata de reconstruir el vínculo social interrumpido por el mal político, restituyendo a los actores sociales su autonomía, asociada a su "capacidad de actuar y hablar", y permitiendo la integración de solicitudes para su posterior respuesta, la cual requiere identificar las valoraciones de los bienes a distribuir para cada contexto. Esto invita a generar procesos dialógicos de distribución que observen la diferenciación contextual de las solicitudes, lo que implica generar propuestas nohomogéneas, sino más bien flexibles y adecuadas en cuanto a la distribución de los bienes disponibles.

En este sentido, se requiere capturar la -relativasignificación contextual de los bienes a distribuir. Ahora bien, se observa que en términos prácticos el paso de lo cualitativo a lo cuantitativo produce una desgarradura interna a la propia idea de distribución por donde puede filtrarse la injusticia. Aquí la exigencia es inventar proporcionalidades que, en lo posible, salven dicha desgarradura de forma histórica y contextual.

\section{Conclusión}

Vista desde Ricoeur, la noción de justicia se basa en la estructura de la moralidad que va de los ideales de vida buena al establecimiento de las normas sociales, pasando por la mediación de las instituciones dentro de sociedades democráticas. Esto implica una relación de complementariedad entre una tradición teleológica y una deontológica, y de algún modo a poner en otro nivel la estéril discusión entre universalismo y contextualismo. Desde este autor, lo recomendable sería construir una dialéctica entre lo universal -la unidad de la razón práctica- y lo contextual -la diversidad de la vida ética- con el objeto de llegar a lo que se puede denominar un juicio en situación, es decir, una decisión sensible al contexto en el cual se va a desarrollar su efecto. En este marco, la forma de la justicia no presenta ningún contenido a priori o una respuesta fija a las solicitudes sociales, sino que más bien el conteni- do de las decisiones refiere a las circunstancias, las tendencias y orientaciones de vida develados en los procesos deliberativos.

A partir de la lectura de la obra de Ricoeur es posible observar una noción de justicia en la que las relaciones morales, jurídicas y políticas no están situadas únicamente bajo la idea de la conformidad con la ley y la legalidad. Más bien, en la noción de justicia de Ricoeur el carácter teleológico de la acción humana es anterior a la constitución de las normas. En definitiva, las capacidades dignas de respeto de todo hombre se actualizan por medio de la institución. De este modo, el valor ético del nivel propiamente político de la institución es la justicia, y su aceptabilidad depende a la vez de las modalidades y grados de participación en la decisión política.

En suma, la justicia de la que habla Ricoeur es, más allá de lo legal, fundamentalmente distributiva, pero al ir más allá de lo económico se ve desmembrada en una serie de esferas donde se requiere establecer la prioridad que ha de darse a cada una de ellas. Ricoeur realiza su planteamiento en contra de entender lo justo independientemente del bien. De ahí el esfuerzo del autor por señalar que la justicia no es puramente "moral", sino que tiene también un significado "ético", a partir de lo cual "lo justo" se sitúa entre "lo legal y lo bueno" (Ricoeur, 1990, p. 35).

Con lo dicho, podemos ver que la noción de justicia implica la consideración por el "otro", y que, correlativamente, la justicia se extiende más allá de la relación "cara a cara". Así, el "vivir bien" no se limita únicamente a las relaciones interpersonales, donde opera la solicitud, sino que se extiende a la vida de las instituciones, lugar de aplicación de la justicia. En este marco, la justicia presenta un rasgo que no está contenido en la noción de solicitud -por el bien del otro-, a saber, la exigencia de igualdad, principio que se convierte en el contenido ético del sentido de justicia. A partir de este análisis encontramos que el núcleo común de la justicia distributiva (relacionada con las transacciones públicas) y de la justicia reparadora (relacionada con las transacciones privadas) es la igualdad -isotés-.

De este modo, para enfrentar dificultad de los repartos desiguales, y desde la noción de igualdad que acompaña a la justicia, Ricoeur plantea, como ya hemos visto, en un primer momento la idea de reparto aritmético igualitario, el cual, a pesar de ser difícil de concebir, no deja de tener aplicación práctica. Para el autor, en las sociedades modernas no ha cesado el campo de aplicación de la igualdad aritmética: igualdad ante la ley, derecho de igual voto, entre otros. No obstante, esta noción de igualdad 
parece no ser suficiente frente a la policontextualidad y diversidad que caracteriza a las sociedades actuales; por cuanto para alcanzar este tipo de igualdad hay que tratar desigualmente a personas en principio iguales, como sucede con políticas que favorecen a algunos respecto de otros en situaciones similares. Así, y frente a la insuficiencia de la igualdad aritmética, Ricoeur trae a colación lo que define a la justicia distributiva, a saber, la igualdad proporcional, que consiste en hacer iguales las relaciones entre una persona y un mérito cada vez (Ricoeur, 1990). Dicho de otro modo, la igualdad proporcional -equidad en este punto-, es a la vida en las instituciones lo que la solicitud es a las relaciones interpersonales.

En este punto, Ricoeur, al apelar a las instituciones públicas -fundamentalmente del Estado-, propone que la justicia exige tener en cuenta de modo preferencial los intereses de los marginados, para compensar el desequilibrio estructural que padecen mediante medidas proporcionales y situacionales. Ahora bien, en todo esto "no se trata de una lógica de la necesidad dialéctica sino de una dramática de la libertad" (Ricoeur, 1990, p. 166), en este sentido, la distribución no solo tiene que ver con los bienes sociales, sino también con el reparto y disminución del sufrimiento que acaparan los males políticos de la sociedad. Lo que aquí se observa es que para Ricoeur la solicitud, que supone el sentido de justicia, no solamente motiva el preferir los intereses de los menos favorecidos de la sociedad, sino que permite captar el "exceso" de vida y de sentido que se da muchas veces y que se observa como injusticia.

Ahora bien, la fijación por los más necesitados no aparece con claridad en la obra de Ricoeur, sin embargo, puede vincularse a su remarcar lo teleológico como búsqueda de una vida buena, como base sobre la que puede construirse una moral bien fundamentada que sea mucho más que meramente procedimental, o reivindicativa, en sentido negativo, de una igualdad vacía.

Hasta aquí, la justicia es una idea que recoge lo mejor del pensamiento de Ricoeur sobre lo justo en su vertiente ética y jurídica, y su lectura significa una oportunidad para replantear las nociones de justicia que operan en nuestras instituciones sociales y las de nuestros organismos de control político.

\section{Bibliografía}

- BEGUÉ, M-F. (2002) "Paul Ricoeur: la poética del símismo". Biblos. Buenos Aires.

- CORTINA, A. (1998) "Ciudadanos del mundo. Hacia una teoría de la ciudadanía". Alianza Editorial, Madrid.

- DE LA MAZA, L.M. (2010) "Actualizaciones del concepto hegeliano de reconocimiento". Separata. Revista VERITAS, N²3. Septiembre.

- LADRIERE, J. (1997) "La ética en el Universo de la Racionalidad". Editions Fides, Québec, Canadá.

- RICOEUR, P. (1990) "Amor y Justicia”. Caparros, Madrid.

- RICOEUR, P. (1993) "Morale, éthique et politique", en Pouvoirs 65, Paris.

- RICOEUR, P. (1996) "Tolérance, intolérance, intolérable", en Lectures I. Gallimard, Francia.

- Ricoeur, P. (1990, 1996) "Sí mismo como otro". México, D.F.: Siglo Veintiuno.

- RICOEUR, P. (1997) "Lo justo". Barcelona : Ed. Jurídica de Chile.

- RICOEUR, P. (2000); "Luniversel et l'historique", en Magazine littéraire 390, Paris.

- RICOEUR, P. (2001) “Lo justo 2". Editorial Trotta, Madrid.

- RICOEUR, P. (2006) "Caminos del reconocimiento"; En: http://www.pdfgratis.org/Paul-Ricoeur-Caminosdel-reconocimiento. 